\title{
New Mathematical Tools for the Study of the DNA Structure
}

\author{
Juan Antonio Nieto, Constanza Celia Nieto-Marín, Nayeli Nieto-Marín, Itzel Nieto-Marín \\ Facultad de Ciencias Fsico-Matemáticas de la Universidad Autónoma, de Sinaloa, Culiacán, Sinaloa, México \\ Email: niet@uas.edu.mx, janieto1@asu.edu,constanza.cnm6@gmail.com,nayel_nm13@hotmail.com,nmitzel@hotmail.com
}

How to cite this paper: Nieto, J.A., Nieto-Marín, C.C., Nieto-Marín, N. and Nieto-Marín, I. (2021) New Mathematical Tools for the Study of the DNA Structure. Journal of Applied Mathematics and Physics, 9, 1896-1903.

https://doi.org/10.4236/jamp.2021.98123

Received: June 30, 2021

Accepted: August 8, 2021

Published: August 11, 2021

Copyright $\odot 2021$ by author(s) and Scientific Research Publishing Inc. This work is licensed under the Creative Commons Attribution International License (CC BY 4.0).

http://creativecommons.org/licenses/by/4.0/

\begin{abstract}
We propose a number of new mathematical tools for the study of the DNA structure. In particular, we establish a connection between the DNA molecule and the Grassmann-Plücker coordinates, which, in both in mathematics and physics, are of great importance.
\end{abstract}

\section{Keywords \\ DNA Structure, (4 + 4)-Dimensions, Grassmann-Plücker Relations}

\section{Introduction}

The present problems emerging from the pandemic of the so-called covid-19 virus [1] have motivated many researches of different areas to participate in searching for a better understanding of their various features. In particular, it becomes attractive to see in deep details how the RNA of the virus interacts with the DNA molecule contained in the nucleus of the cells [2]. In this sense, it may be helpful to introduce new mathematical tools for the study of the DNA structure.

In this work, we show that the so-called Grassmann-Plücker coordinates [3] may be connected with the DNA structure. We argue that this link may be helpful for a building, a more complete bridge between the many mathematical and physical scenarios related with Grassmann-Plücker coordinates and the DNA molecule.

It turns out that Grassmann-Plücker coordinates are one of the key concepts to study chirality in some chemical molecules such as tartaric acid. In fact, this kind of molecule admits a description in terms of three distinct molecular forms, known as dextro, levo, and nueso. Moreover, this can be illustrated by tetrahedron around the carbon atom (see Ref. [4] for details). Here, we are concerned 
with the fact that the chirality of this kind of chimical molecule eventually leads to the concept of chirotope of oriented matroid theory [4] (see also Refs. [5] [6] and [7]). In turn, the chirotopes can be understood as generalization of the Grassmann-Plücker coordinates. Specifically, one considers the chirality of the tartaric acid in terms of a chirotope which assigns a + or - sign whether the four neighbors in a certain order have right-handed or left-handed orientation. Thus, the associated chirotope may be regarded as a mathematical-chemical concept of chirality (see Refs. [4] [5] [6] and [7] for details). Our novel contribution in the context of these developments is that we associate the Grassmann-Plücker coordinates (chirotopes) with the DNA structure.

Technically, this brief note is organized as follows. In Section 2, we start with a brief review of the DNA structure. While in Section 3, we briefly comment about the Grassmann-Plücker coordinates. In this way in Section 4, we establish a connection between the Grassmann-Plücker coordinates and the DNA structure. We finish this work giving a number of comments about a possible link between the DNA and oriented matroid theory [4] (see also Refs. [8]-[14]), $(4+4)$-dimensions [15] [16] [17] [18] and surreal numbers [19] [20] (see also Refs. [21] and [22]).

\section{A Brief Review of the DNA Structure}

In general, one can say that the DNA structure is a nucleic acid that lives in the interior of the nucleus of a cell, and it is one of the four major groups of biological macromolecules. In fact, all nucleic acids are made up of nucleotides. In DNA, each nucleotide is made up of three parts: a sugar molecule, a phosphate group, and a nitrogenous base. Moreover, the DNA structure forms part of the interior of the cell as a mass of genetic material known as chromatin and by a special compactification of the DNA structure during de cell division a special compactification of the DNA structure a chromosome is formed which of course also live in the interior of a nucleus. The well known form of the DNA is two strands of double helix stair shape with 4-bases, adenine (A), thymine (T), cytosine $(\mathrm{C})$ and guanine $(\mathrm{G})$ forming the stairs rungs and sugar and phosphate forming the stairs poles. The interesting thing is that, because is chemical properties, adenine can only be combined with thyamine and cytosine with guanine (see Ref. [2] and references therein). Schematically, we can consider these 4 options in the form;

$$
\begin{aligned}
& A-T, \\
& T-A, \\
& C-G, \\
& G-C .
\end{aligned}
$$

We observe that the bases on the two strands of a DNA structure are complementary or dual. The reason for this seems to be that adenine and thymine form two hydrogen bonds, while the cytosine and guanine form three hydrogen bonds. 
Just to have an idea, in a nucleus of human cell there are 46 chromosomes and roughly speaking each of the chromosomes is made of $10^{9}$ pair of bases, according to (1). Moreover, a gen is defined as a sector of the DNA with various numbers of stair rungs, a estimate could be around $10^{4}$ stairs rungs. This means that each gen is made of about $10^{5}$ pair bases. Of course, each chromosome and each living being have many possible variants.

\section{Brief Comments about the Grassmann-Plücker Coordinates}

Consider a matrix $A$ of the form

$$
A=\left(\begin{array}{llll}
a & b & c & d \\
e & f & g & h
\end{array}\right) .
$$

This is a $4 \times 2$-matrix, that is a matrix with 4 -column and 2-raw. Instead of (2), one may introduce the notation

$$
v_{i}^{\mu} \equiv\left(\begin{array}{llll}
v_{1}^{1} & v_{1}^{2} & v_{1}^{3} & v_{1}^{4} \\
v_{2}^{1} & v_{2}^{2} & v_{2}^{3} & v_{2}^{4}
\end{array}\right) .
$$

The identification of each term in (1) and (2) is straightforward. For instance one may see that $v_{2}^{3}=g$. Now we introduce the quantity

$$
F^{\mu \nu} \equiv \varepsilon^{i j} v_{i}^{\mu} v_{j}^{v}=-F^{v \mu}
$$

Here, we are using the convention in tensor analysis that repeated indices mean a sum. Moreover, the only non-vanishing terms of the $\mathcal{\varepsilon}$-symbol $\varepsilon^{i j}=-\varepsilon^{j i}$ are

$$
\begin{aligned}
\varepsilon^{12} & =1, \\
\varepsilon^{21} & =-1 .
\end{aligned}
$$

Using the properties of $\varepsilon^{i j}$ it is not difficult to show that $F^{\mu v}$ satisfies the Grassmann-Plücker relations

$$
F^{\mu[v} F^{\alpha \beta]}=0,
$$

where $[v \alpha \beta]$ means totally antisymmetric. Indeed, one can prove that (4) is satisfies if and only if (6) holds. Of course, the algorithm can be generalized in several fronts. The reference [11] provides a number of generalizations of this result. In particular, if one introduces the totally antisymmetric $\mathcal{E}$-symbol with $k$ indices $\varepsilon^{i_{1} \cdots i_{k}}$ one can write the Grassmann-Plücker coordinates as

$$
F^{\mu_{1} \cdots \mu_{k}} \equiv \varepsilon^{i_{1} \cdots i_{k}} v_{i_{1}}^{\mu_{1}} \cdots v_{i_{k}}^{\mu_{k}},
$$

which implies

$$
F^{\mu_{1} \cdots\left[\mu_{k}\right.} F^{\left.\nu_{1} \cdots v_{k}\right]}=0 .
$$

Of course, one must require that the dimension where the indices $\mu, v, \cdots$ etc. "live" is greater than $k$.

\section{Link between the Grassmann-Plücker Coordinates and the DNA}

At first sight it seems an impossible task to relate (1) with (4). But in what fol- 
lows we shall show step by a step that this is in fact possible. Let us start rewriting the squematic relation (1) as

$$
\begin{aligned}
& \mathrm{A}^{+} \rightarrow \mathrm{T}^{-}, \\
& \mathrm{T}^{+} \rightarrow \mathrm{A}^{-}, \\
& \mathrm{C}^{+} \rightarrow \mathrm{G}^{-}, \\
& \mathrm{G}^{+} \rightarrow \mathrm{C}^{-} .
\end{aligned}
$$

In this form one is considering that the letters $\mathrm{A}^{+}, \mathrm{T}^{+}, \mathrm{C}^{+}$and $\mathrm{G}^{+}$are in the right and $\mathrm{A}^{-}, \mathrm{T}^{-}, \mathrm{C}^{-}$and $\mathrm{G}^{-}$are in the left. Instead of letters one can equally put numbers in the form

$$
\begin{aligned}
& 1^{+} \rightarrow 2^{-}, \\
& 2^{+} \rightarrow 1^{-}, \\
& 3^{+} \rightarrow 4^{-}, \\
& 4^{+} \rightarrow 3^{-} .
\end{aligned}
$$

For further change, let us consider the combinations

$$
\begin{aligned}
& 1^{+} \searrow 1^{-}, \\
& 2^{+} \nearrow 2^{-} \\
& 3^{+} \searrow 3^{-}, \\
& 4^{+} \nearrow 4^{-} .
\end{aligned}
$$

So, in this way we have identified two well defined matrices, namely

$$
\left(\begin{array}{lll}
1^{+} & \searrow 1^{-} \\
2^{+} & \nearrow 2^{-}
\end{array}\right)
$$

and

$$
\left(\begin{array}{lll}
3^{+} & \searrow 3^{-} \\
4^{+} & \nearrow & 4^{-}
\end{array}\right)
$$

One can even write (10) and (11) in more abstract terms. Consider the definitions

$$
\begin{aligned}
& x^{1+} \equiv 1^{+}, x^{2+} \equiv 2^{+}, x^{3+} \equiv 3^{+} \text {and } x^{4+} \equiv 4^{+}, \\
& x^{1-} \equiv 1^{-}, x^{2-} \equiv 2^{-}, x^{3-} \equiv 3^{-} \text {and } x^{4-} \equiv 4^{-} .
\end{aligned}
$$

With this new notation we can rewrite the matrices (12) and (13) in the form

$$
v_{i}^{\mu} \equiv\left(\begin{array}{llll}
x^{1+} & x^{1-} & 0 & 0 \\
x^{2+} & x^{2-} & 0 & 0
\end{array}\right)
$$

and

$$
v_{a}^{\mu} \equiv\left(\begin{array}{cccc}
0 & 0 & x^{3+} & x^{3-} \\
0 & 0 & x^{4+} & x^{4-}
\end{array}\right) .
$$

respectively. This suggests to write a complete matrix of the form

$$
v_{A}^{\mu} \equiv\left(\begin{array}{llll}
x^{1+} & x^{1-} & 0 & 0 \\
x^{2+} & x^{2-} & 0 & 0 \\
0 & 0 & x^{3+} & x^{3-} \\
0 & 0 & x^{4+} & x^{4-}
\end{array}\right) .
$$


Now, we would like to argue that main conditions in the bases of the DNA can be found in the expression (17). The first observation is that (17) has the form of two diagonal $2 \times 2$-diagonal matrix. This means that there are not mixture between the bases (A, T) and (C, G) bases. Now the specific combination in (1) can be associated with the requirement that determinance of such a block-diagonal matrice is different from zero. Let us be more specific, if

$$
\operatorname{det} M \equiv \operatorname{det}\left(\begin{array}{ll}
x^{1+} & x^{1-} \\
x^{2+} & x^{2-}
\end{array}\right) \neq 0,
$$

then only the first two combinations in (1) are possible, that is, one has A-T, with $\mathrm{A}$ and $\mathrm{T}$ in the first and the second column, respectively and also $\mathrm{T}-\mathrm{A}$, with $\mathrm{T}$ and $\mathrm{A}$ in the first and the second column respectively. Similarly, if

$$
\operatorname{det} N \equiv\left(\begin{array}{ll}
x^{3+} & x^{3-} \\
x^{4+} & x^{4-}
\end{array}\right) \neq 0,
$$

then only the last two combinations in (1) are possible that is C-G, with C and G in the first and the second column, respectively and also G-C, with G and C in the first and the second column respectively. On the other hand if we call $V$ the space generated by (12), then one must write

$$
V=M \otimes N,
$$

where the symbol $\otimes$ denotes direct product, indicating that there is not mixture between the bases $(\mathrm{A}, \mathrm{T})$ and $(\mathrm{C}, \mathrm{G})$.

Thus, one can introduce two dual Grassmann-Plücker coordinates

$$
\mathcal{F}^{\mu v} \equiv \varepsilon^{i j} v_{i}^{\mu} v_{j}^{v}=-\mathcal{F}^{v \mu}
$$

and

$$
{ }^{*} \mathcal{F}^{\mu \nu} \equiv \varepsilon^{a b} v_{a}^{\mu} v_{b}^{v}=-{ }^{*} \mathcal{F}^{\nu \mu},
$$

with the dual relation

$$
{ }^{*} \mathcal{F}^{\mu v}=\frac{1}{2} \varepsilon_{\alpha \beta}^{\mu v} \mathcal{F}^{\alpha \beta} .
$$

Assuming that $\mathcal{F}^{\mu \nu}$ cannot always be written as (21) one obtains an extended notion of the Grassmann-Plücker coordinates which lead eventually to one of the possible definitions of oriented matroids [3] and [4].

\section{Final Remarks}

Summarizing, we have shown that the so-called Grassmann-Plücker coordinates [3] may be connected with the DNA structure. Our main argument is that the 4 options of the DNA structure in (1) can be properly expressed in matrix form, which properties can be naturally put in a frame of Grassmann-Plücker coordinates. Moreover, to see one possible implication of this result, by noting that the adenine and thymine form two hydrogen bonds, while the cytosine and guanine form three hydrogen bonds, we prove that the bases on the two strands of a DNA structure are complementary or dual, in agreement with the dual property 
of the Grassmann-Plücker coordinates.

The formalism developed in this work may motivate to consider different routes for further research in the study of the DNA structure. For instance, looking at the coordinates $x^{1+}, x^{2+}, x^{3+}, x^{4+}$ and $x^{1-}, x^{2-}, x^{3-}, x^{4-}$ in (14) one may have the idea that geometrically one can associate the line element

$$
\begin{aligned}
\mathrm{d} s^{2}= & +\left(\mathrm{d} x^{1+}\right)^{2}+\left(\mathrm{d} x^{2+}\right)^{2}+\left(\mathrm{d} x^{3+}\right)^{2}+\left(\mathrm{d} x^{4+}\right)^{2} \\
& -\left(\mathrm{d} x^{1-}\right)^{2}-\left(x^{2-}\right)^{2}-\left(x^{3-}\right)^{2}-\left(x^{4-}\right)^{2},
\end{aligned}
$$

which in the context of special relativity refers as a theory of four space and four times ((4+4)-dimensional spacetime) background (see Refs. [15] [16] [17] [18]) and references therein).

On the other hand, since the Grassmann-Plücker is connected to many mathematical and physical scenarios, the bridge proposed in this work may help to better understand the DNA structure. Among such many options let us choose oriented matroid theory, but in principle Grassmann-Plücker relations are linked to topology theory among others. As we mentioned in the introduction, this mathematical notion has already been considered to describe some aspect of chemical molecules (see Ref. [4]). And in fact, it turns out that the Grassmann-Plücker coordinates can be used to introduce one of the many possible definitions of oriented matroids [4]. Indeed, we would like to emphasize that a generalization of the Grassmann-Plücker relations can be used to define the so-called chirotopes, which is a key concept in oriented matroid theory (see also Refs. [8]-[14] and references therein). In this sense we believe that the present work may be useful for finding a more formal link between chirotopes and the DNA structure. It is worth mentioning that in oriented matroid theory one finds the mathematical concept of mutation [4]. Roughly speaking this means that, spontaneously, a basis in a given matroid changes sign. We may ask: is this curious coincidence of matroid mutation be related with the mutation dynamics in the DNA?

If one thinks in the matrices (18) and (19) one observes that such matrices can be considered as elements of the so-called $\operatorname{SL}(2, R)$ group. This is a Lie group which the corresponding Casimir operator $k(k-1)$ has an interesting relation with the so called surreal number theory. In fact, the lowest eigenvalues satisfy the formula

$$
k(k-1)+\frac{3}{16}=0 .
$$

The solutions of this equation are two of the surreal numbers $\frac{1}{4}$ and $\frac{3}{4}$. If one further extend this argument to higher eigenvalues one obtains the equation

$$
(k(k-1))^{2}+\frac{11}{32} k(k-1)+\frac{105}{64^{2}}=0,
$$

which lead to the two equations

$$
k(k-1)=-\frac{7}{64}
$$


and

$$
k(k-1)=-\frac{15}{64}
$$

Thus, one finds that (27) admits the solutions $\frac{1}{8}$ and $\frac{3}{8}$, while (28) leads to the values $\frac{5}{8}$ and $\frac{7}{8}$. All these values $\frac{1}{4}, \frac{3}{4}, \frac{1}{8}, \frac{3}{8}, \frac{5}{8}$ and $\frac{7}{8}$ are of the type $\frac{m}{2^{n}}$, with $m$ an integer and $n$ is a positive natural. It turns out that the numbers $\frac{m}{2^{n}}$ are the so called dyadic rationals and are the key numbers in the structure of the so called surreal numbers [19] and [20] (see also Refs. [21] and [22]).

Of course, our algorithm (15)-(17) corresponds to a very simplified sector of genome which is very long stretch of DNA. But the idea will be to extend the present work to the complete genome contained inside the nucleus of a cell. Eventually, this may help to have better understanding of the genome dynamics.

\section{Acknowledgements}

JA Nieto would like to thank his students of gravitation III and general relativity and advance cosmology for helpful comments. This work was partially supported by PROFAPI 2013.

\section{Conflicts of Interest}

The authors declare no conflicts of interest regarding the publication of this paper.

\section{References}

[1] Coronavirus Disease (COVID-19) Pandemic. World Health Organization. https://www.who.int

[2] Watson, J.D. and Berry, A. (2009) DNA: The Secret of Life (Kindle of Knopf Edition).

[3] Bokowski, J. and Sturmfels, B. (1980) Computational Synthetic Geometry. Springer-Verlag, Berlin.

[4] Björner, A., Las Vergnas, M., Sturmfels, B., White, N. and Ziegler, G.M. (1993) Oriented Matroids. Cambridge University Press, Cambridge.

[5] Crippen, G.M. and Havel, T.F. (1988) Distance Geometry and Molecular Conformation. Research Studies Press, Taunton, 541.

[6] Dreiding, A., Dress, A.W.M. and Haegi, H.A. (1982) Classification of Mobile Molecules by Category Theory. Studies in Physical and Theoretical Chemistry, 23, 39.

[7] Klin, M.H., Tratch, S.S. and Zefirov, N.S. (1990) 2D-Configurations and Clique-Cyclic Orientations of Graphs L(Kp). Reports in Molecular Theory, 1, 149.

[8] Nieto, J.A. (2004) Matroids and p-Branes. Advances in Theoretical and Mathematical Physics, 8, 177-188. https://doi.org/10.4310/ATMP.2004.v8.n1.a4

[9] Nieto, J.A. (2006) Oriented Matroid Theory as a Mathematical Framework for M-Theory. Advances in Theoretical and Mathematical Physics, 10, 747-757. 
https://doi.org/10.4310/ATMP.2006.v10.n5.a5

[10] Nieto, J.A. (2004) Searching for a Connection between Matroid Theory and String Theory. Journal of Mathematical Physics, 45, 285.

https://doi.org/10.1063/1.1625416

[11] Nieto, J.A. (2014) Phirotopes, Super p-Branes and Qubit Theory. Nuclear Physics B, 883, 350-372. https://doi.org/10.1016/j.nuclphysb.2014.04.001

[12] Nieto, J.A. and Marn, M.C. (2000) Matroid Theory and Chern-Simons. Journal of Mathematical Physics, 41, 7997. https://doi.org/10.1063/1.1319518

[13] Nieto, J.A. (2013) Qubits and Oriented Matroids in Four Time and Four Space Dimensions. Physics Letters B, 718, 1543-1547.

https://doi.org/10.1016/j.physletb.2012.12.034

[14] Nieto, J.A. (2010) Qubits and Chirotopes. Physics Letters B, 692, 43-46. https://doi.org/10.1016/j.physletb.2010.07.010

[15] Nieto, J.A. and Espinoza, M. (2016) Dirac Equation in Four Time and Four Space Dimensions. International Journal of Geometric Methods in Modern Physics, 14, Article ID: 1750014. https://doi.org/10.1142/S0219887817500141

[16] Nieto, J.A. and Madriz-Aguilar, J.E. (2019) Aspects of $(4+4)$-Kaluza-Klein Type Theory. Physica Scripta, 94, Article ID: 115303.

https://doi.org/10.1088/1402-4896/ab2d96

[17] Nieto, J.A. (2005) Towards an Ashtekar Formalism in Eight Dimensions. Classical and Quantum Gravity, 22, 947-955. https://doi.org/10.1088/0264-9381/22/6/004

[18] Nieto, J.A. (2016) Alternative Self-Dual Gravity in Eight Dimensions. Modern Physics Letters A, 31, Article ID: 1650147.

https://doi.org/10.1142/S0217732316501479

[19] Conway, J.H. (1976) On Number and Games (London Mathematical Society Monographs). Academic Press, Cambridge.

[20] Gonshor, H. (1986) An Introduction to the Theory of Surreal Numbers (London Mathematical Society Lectures Notes Series, Vol. 110). Cambridge University Press, Cambridge. https://doi.org/10.1017/CBO9780511629143

[21] Nieto, J.A. (2018) Duality, Matroids, Qubits, Twistors, and Surreal Numbers. Frontiers in Physics, 6, Article No. 106. https://doi.org/10.3389/fphy.2018.00106

[22] Avalos-Ramos, C., Félix-Algandar, J.A. and Nieto, J.A. (2020) Dyadic Rationals and Surreal Number Theory. IOSR Journal of Mathematics (IOSR-JM), 16, 35. 